\title{
Towards Appliance Usage Prediction for Home Energy Management
}

\author{
Ngoc Cuong Truong, Long Tran-Thanh, Enrico Costanza and Sarvapali D. Ramchurn \\ Agent, Interaction and Complexity Group \\ Electronics and Computer Science \\ University of Southampton, UK \\ \{nct1g10, Itt08r, ec, sdr\}@ecs.soton.ac.uk
}

\begin{abstract}
In this paper, we address the problem of predicting the usage of home appliances where a key challenge is to model the everyday routine of homeowners and the inter-dependency between the use of different appliances. To this end, we propose an agent based prediction algorithm that captures the everyday habits by exploiting their periodic features. We demonstrate that our approach outperforms existing methods by up to $40 \%$ in experiments based on real-world data from a prominent database of home energy usage.
\end{abstract}

\section{Categories and Subject Descriptors}

I.2.6 [Artificial Intelligence]: Learning

\section{Keywords}

Usage Prediction, Home Energy Management

\section{INTRODUCTION}

In the face of dwindling fossil fuels, an ageing electricity distribution infrastructure, and the adverse effects of high levels of green house gasses on climate change, the problem of generating affordable and clean electricity reliability is one of the greatest challenges of this century [1]. Now, to make the use of the electrical devices in the home more efficient, and thus, to reduce both carbon emissions and cost, a set of agent based demand side management techniques have recently been introduced to optimise the schedule of loads [4]. However, these techniques typically do not take into account the homeowner's preferences in their optimisation, and ignore inter-dependencies between the usage of different appliances.. Thus, the main challenge is to predict the energy consumption activities of homeowners, so that the agent can design optimal schedules by planning ahead the electricity usage that meets the human's preferences.

Against this background, we propose a novel approach to predicting the energy consumption of different home appliances, that takes into account both the human routine activities and the inter-dependency between appliances. To do so, we rely on the common assumption that human behaviour follows a certain cyclic pattern [2]. Based on this, we build a model that exploits this cyclic behaviour. To handle the inter-dependency between the appliances, we use the

Copyright is held by the author/owner(s). ACM X-XXXXX-XX-X/XX/XX. episode generation Hidden Markov model (EGH) [5] to efficiently identify the patterns that form the inter-dependency between the usage of the appliances. By putting the two models together, we demonstrate that our approach outperforms the state-of-the-art, that only focus on either human behaviour detection on inter-dependency pattern identification. We formalise our problem scenario in Section 2. Section 3 evaluates the algorithm and analyses the results.

\section{PREDICTING THE USAGE ACTIVITIES OF APPLIANCES}

We first describe the formalisation of our problem in Section 2.1, then introduce our algorithm in Section 2.2.

\subsection{Model Description}

We assume that we have a finite set of consumer activities, where different types of activities are distinguished by labels $l \in L$. An activity profile of label $l a_{l, t}$ is a tuple $\langle t, l, n\rangle$, composed of a time step $t$ (measured in days), a label $l$ and number of usage $n$, that denotes the number of occurrences of label $l$ on day $t$. Let $x_{t}=\left\langle a_{1, t}, a_{2, t}, \ldots, a_{L, t}\right\rangle$ denote the usage profile of day $t$ that contains the information about the usage of each label $l \in L$ on day $t$. The appliance usage history $h_{t}$ of time slot $t$ is the sequence $h_{t}=\left\{x_{1}, x_{2}, \ldots, x_{t}\right\}$. Our goal is to estimate $x_{t+1}$ for any $t>0$, given $h_{t}$.

\subsection{The Prediction Algorithm}

As mentioned earlier, the foundations of our prediction algorithm rely on the EGH method. However, as EGH is not designed for detecting human activities, we tailor the model to fit our settings by exploiting the periodic features of the human everyday routine. We build the inter-dependency model by relying on the EGH approach described by Srivatsa et al. [5]. Based on these models, we then construct a mixture model of the significant episodes (i.e. sets of possible inter-dependency rules) in order to calculate the probability of activities' occurrence in Section 2.2.2. Finally, Section 2.2.3 focuses on the prediction model in detail.

\subsubsection{The Human Routine Model}

We assume that human behaviour in home energy usage follows a weekly cycle. More formally, let $K$ denote the number of occurrences of the target activity type $l$ on the specific day $d$ of the week in the activity usage history $h_{t-1}$. Thus, for each label $l$ and the prediction day of the week $d$, from the original training dataset $D$, we extract a training set $D_{l, d}=\left\{X_{i}\right\}_{i=1}^{K}$, where $X_{i}=\left\langle x_{t_{i}-7}, \ldots, x_{t_{i}-1}>\right.$ is the weekly preceding window of activities from $x$ that imme- 

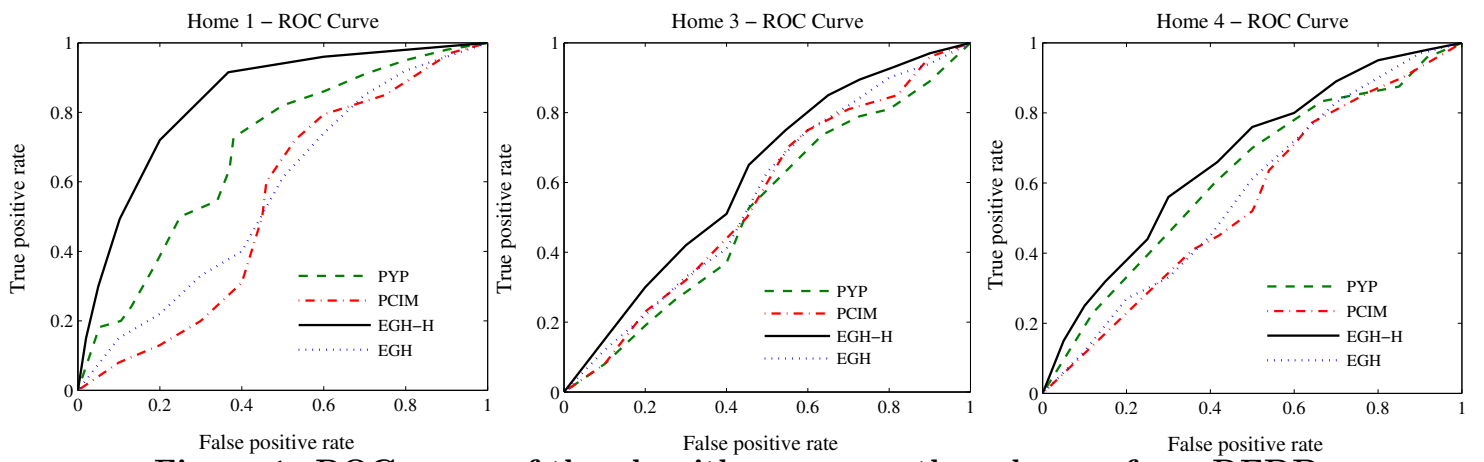

Figure 1: ROC curve of the algorithms run on three homes from REDD.

diately preceded the $i^{t h}$ occurrence of $l$ in $x$, and $t_{i}$ is the time that the target activity type $l$ occurred at the $i^{\text {th }}$ in the activity sequence. By doing so, we can reduce the computational costs and also improve the quality of prediction (as we will demonstrate later in Section 3).

\subsubsection{The Mixture Model}

Supppose that for a given training data set $D_{l, d}=\left\{X_{i}\right\}_{i=1}^{K}$, we have calculated a set of significant episodes, denoted as $F^{s}=\left\{\alpha_{1}, \ldots, \alpha_{J}\right\}$, and each HMM $H_{\alpha_{j}}$ of episode $\alpha_{j}$. To model the effect of this joint influence, we compute a mixture model $\Lambda_{l}$ (i.e. a combination of probabilistic processes) of the significant episodes' HMMs. The likelihood function of the training dataset $D$ under a mixture model $\Lambda_{l}$ is: $P\left[D \mid \Lambda_{l}\right]=\prod_{i=1}^{K} P\left[X_{i} \mid \Lambda_{l}\right]=\prod_{i=1}^{K}\left(\sum_{j=1}^{J} \theta_{j} P\left[X_{i} \mid H_{\alpha_{j}}\right]\right)$, where $\theta_{j}, j=1 . . J$ are the mixture coefficients of $\Lambda_{l}$ (with $\theta_{j} \in[0,1]$ for all $j$, and $\left.\sum_{j=1}^{J} \theta_{j}=1\right)$. We use the Expectation Maximisation (EM) algorithm to estimate the set of mixture coefficients of the mixture model $\Lambda_{l}$.

\subsubsection{The Prediction Model}

Let $t$ denote the current time. For the set of target activity labels $l \in$, we want to predict their occurrences in the next day, $t+1$. As we are mainly interested in occurrences of recent activities of the users, therefore, we construct a 7 length window of activities from the weekly period $[t-7, t]$. We then estimate the likelihood of this recent activity sequence, given the mixture model, $\Lambda_{l}=\left\{\left(\alpha_{j}, \theta_{j}\right)\right\}_{j=1, \ldots, J}$, that is obtained from the training phase.

\section{EMPIRICAL EVALUATION}

Given the prediction model, we now demonstrate how our algorithm outperforms a set of benchmark algorithms, including Pitman-Yor Process (PYP), the piece--wise constant conditional intensity model (PCIM) [3]), and the original EGH method. Here, we perform the algorithms on the realworld REDD dataset ${ }^{1}$ (see Section 3.1). We also compare the average running time of the algorithms in Section 3.2.

\subsection{Performance on REDD Data}

In overall, our method outperforms other state-of-the-art by up to $40 \%$, based on the $F$-score measurement. In particular, it is better than PYP, EGH, and PCIM by approximately $73 \%, 40 \%$, and $75 \%$ on average, respectively. From the figure of the receiver operating characteristic (ROC) curve (Figure 1), we can see that our algorithm dominates

\footnotetext{
${ }^{1}$ http://redd.csail.mit.edu/
}

all the others. In particular, the area under the curve (AUC) of EGH-H in home 1 is 0.84 , while the AUC value for PYP, EGH, and PCIM is $0.68,0.56$, and 0.53 , respectively. We can also observe that since data from homes 3 and 4 is less detailed, all the algorithms provides worse performance, compared to themselves in home 1 . However, our algorithm still dominates the benchmark approaches.

\subsection{Average Running Time of the Algorithms}

We run the algorithms on an $\operatorname{Intel}(\mathrm{R}) \mathrm{Xeon}(\mathrm{R})$ computer (64-bit operating system) with $2.67 \mathrm{GHz}$ and 12GB. We can observe that on average, our algorithm is $1504.78,119.3$, and 151.19 times faster than PYP, PCIM, and EGH on average.

\section{CONCLUSIONS}

We proposed EGH-H, the algorithm that addresses human behaviour prediction within the energy management domain. We also demonstrated through extensive evaluations, using real-world data taken from the REDD, that our algorithm outperforms state-of-the-art methods by up to $40 \%$ in prediction accuracy. As a result, our work could potentially form an efficient solution to real-world home energy management systems, where usage predictions are needed to optimally schedule the electrical consumption of the home. In addition, an improved version is described in [6].

\section{REFERENCES}

[1] Department of Energy \& Climate Change. Smarter Grids : The Opportunity. (December), 2009.

[2] M. C. González, C. A. Hidalgo, and A. Barabási. Understanding individual human mobility patterns. Nature, 453(7196):779-82, June 2008.

[3] A. Gunawardana, C. Meek, and P. Xu. A Model for Temporal Dependencies in Event Streams. NIPS, 2011.

[4] S. D. Ramchurn, P. Vytelingum, A. Rogers, and N. Jennings. Agent-Based Control for Decentralised Demand Side Management in the Smart Grid. In $A A M A S$, pages $5-12,2011$.

[5] L. Srivatsan, P. Sastry, and K. Unnikrishnan. Discovering frequent episodes and learning hidden markov models: A formal connection. IEEE Trans. on Knowledge and Data Eng., 17(11), 2005.

[6] N. C. Truong, J. McInerney, L. Tran-Thanh, E. Costanza, and S. Ramchurn. Forecasting multi-appliance usage for smart home energy management. In Proc. of IJCAI, 2013. 